
\title{
S Research S Suare
}

\section{The Rice Blast Resistance Gene Pid Family has been Strictly Diverged into Indica and Japonica Subspecies}

Ruipeng Chai

South China Agricultural University

Jinyan Wang

South China Agricultural University

Xing Wang

South China Agricultural University

Jianqiang Wen

South China Agricultural University

Xuemei Ye

South China Agricultural University

Yaling Zhang

South China Agricultural University

Yongxiang Yao

South China Agricultural University

Jianfu Zhang

Fujian Academy of Agricultural Sciences

Yihua Zhang

Fujian Academy of Agricultural Sciences

Ling Wang

South China Agricultural University

Qinghua Pan ( $\triangle$ panqh@scau.edu.cn )

South China Agricultural University https://orcid.org/0000-0002-0767-4089

\section{Original article}

Keywords: Oryza sativa, Pyricularia oryzae, indica type resistance gene, japonica type resistance gene, lineage-based divergence

Posted Date: May 4th, 2021

DOI: https://doi.org/10.21203/rs.3.rs-430464/v1 
License: (c) (i) This work is licensed under a Creative Commons Attribution 4.0 International License. Read Full License 


\section{Abstract}

Background: Rice blast (causative agent the fungus Pyricularia oryzae) represents a major constraint over the productivity of one of the world's most important staple foods. Genes encoding resistance have been identified in both the indica and japonica subspecies genepools, and combining these within new cultivars represents a rational means of combating the pathogen.

Results: In this research, a deeper allele mining was carried out on Pid-2, Pid-3, and Pid-4 by their specific FNP markers in the three panels consisting of 70 indica and 58 japonica cultivars. Within Pid-2, three functional and one non-functional alleles were identified; the former were only identified in indica type entries. At Pid-3, four functional and one non-functional alleles were identified, and once again, all of the former were present in indica type entries. However, the pattern of variation at Pid-4 was rather different: here, the five functional alleles uncovered were dispersed across the japonica type germplasm. Among all the 12 candidate functional alleles, both Pid2-ZS and Pid3-ZS were predominant.

Conclusions: Variation in both Pid-2 and Pid-3 appears to have evolved in response to pathogen pressure exerted on indica type cultivars, while that in Pid-4 reflects the interaction between the fungus and the host in japonica type crops. Owning to the founder lineage, 'Zhenzhuai 11-ZS97', rather limited genotypes of the Pidfamily have been effective in both indica and japonica rice groups, of which Pid2-ZS+Pid3-ZS is present in a large proportion of Chinese indica cultivars released since the 1960s.

\section{Background}

Rice (Oryza sativa), a crop domesticated in Asia but now cultivated worldwide, is used as a staple food for half of mankind (Huang et al. 2012; Zhang et al. 2016; Du et al. 2017; Wang et al. 2018; Lv et al. 2020). As a result of two major and independent domestication events, two subspecies have been recognized, namely ssp. indica and ssp. japonica (Huang et al. 2012; Zhang et al. 2016; Choi \& Purugganan 2018; Wang et al. 2018). The two genepools have differentiated over time through them been grown in distinct eco-geographical environments, and have diverged with respect to both the structure of the genome and their gene content (Huang et al. 2012; Ouyang \& Zhang 2013; Zhang et al. 2016; Du et al. 2017; Wang et al. 2018). Introgression from one gene pool to the other is seen as a useful strategy for increasing the crop's genetic diversity.

One of the major pathogens of the crop is the fungus Pyricularia oryzae Cavara (syn. Magnaporthe oryzae Couch), the causative agent of the damaging disease rice blast (Dean et al. 2012; Liu and Wang 2016; Zhang et al. 2017, 2019; Zhao et al. 2018; Huang et al. 2021). A wealth of genes determining resistance to this pathogen has supported the success of using breeding to provide a sustainable means of mitigating the damage caused by blast (He et al. 2012; Zhai et al. 2014; Deng et al. 2017; Zhang et al. $2017,2019)$. The genetics of resistance largely follows the gene-for-gene principle, involving an interaction between a host's resistance gene and a matching avirulence gene in the pathogen (Flor 1971; Ebbole 2007; Wu et al. 2014; Zhang et al. 2015). As a result, following the mutation of matching 
avirulence genes, major gene-based resistances are prone to rapid breakdown. That in turn enable it to create a new genotype of resistance gene to overcome the emerged new race with its new resistance specificity (Zeigler et al. 1995; Wu et al. 2014; Zhang et al. 2015; Wang et al. 2017; Zhang et al. 2017, 2019; Zhao et al. 2018; Huang et al. 2021). New resistance specificities can be generated by mutations to a resistance gene's coding sequence, either in the form of a single nucleotide polymorphic (SNP) and even multiple nucleotide polymorphic mutations (so-called insertion/deletion, InDel) (Bryan et al. 2000; Lin et al. 2007; Liu et al. 2007; Fukuoka et al. 2009; Hua et al. 2012; Zhai et al. 2011, 2014;Zhao et al. 2018); where such changes result in an altered reaction to the pathogen, the mutation is referred to as a functional nucleotide polymorphism (FNP) (Hua et al. 2012; Zhai et al. 2011, 2014). As per the gene-forgene principal, it is envisaged that the stronger arms race led to more FNPs emerged in any given resistance gene (Wu et al. 2014; Zhang et al. 2015; Wang et al. 2017; Zhang et al. 2017, 2019; Zhao et al. 2018; Huang et al. 2021). Searching for FNPs in established host cultivars is considered as an efficient way of identifying the novel resistances required for crop improvement (Li et al. 2019; Teerasan et al. 2019; Tian et al. 2020; Xiao et al. 2020; Zhou et al. 2020).

At least 100 major genes encoding resistance to $P$. oryzae are known, an increasing number of which have been isolated (Liu and Wang 2016; Zhao et al. 2018). Among the latter are the three genes Pid-2, -3 and -4 , present as a cluster on chromosome 6 (here after the Pid family) (Chen et al. 2006, 2018; Shang et al. 2009). The objective of the present study was to devise a set of reliable FNP markers based on variations in genomic sequences of the Pid family, and to use these to exploit the extent of allelic variation available in rice germplasm. A particular focus was to reveal the genetic basis underlying resistance gene divergence between indica and japonica subspecies.

\section{Methods}

\section{Development of FNP markers}

The DNA sequences of Pid-2 (FJ915121.1), Pid-3 (FJ745364.1), and Pid-4 (MG839283.1) present in Digu as well as in a number of reference cultivars were retrieved from GenBank (http://www.ncbi.nim.nih.gov/), and each set of alleles was aligned using Multalin (http://multalin.toulouse.inra.fr/multalin/). Two types of FNPs were targeted, the first represented either a functional or a nonfunctional haplotype, and the second an individual allele. An interval sequence of each candidate FNP was subjected to various marker designations including CAPS (cleaved amplified polymorphism sequences), and dCAPS (derived CAPS) using dCAPS Finder 2.0 (http://helix.wustl.edu/dcaps/dcaps.html). The necessary primer sequences were generated using Primer3 software (https://primer3.ut.ee). As for sequence largely diverged intervals, PCR was drove by triple and/or degenerate primers (Table S1; Zeng 2020; Zhang 2020).

\section{Marker verification}


The FNP assays were validated by testing a larger set of control cvs (so-called CKs), namely Digu (DIG), Tetep (TTP), C039, Zhenshan97 (ZS97), Tadukan (TDK), Nipponbare (NPB), Koshihikari (KSH) and Shennong265 (SN265). Each $20 \mu \mathrm{L}$ PCR contained $0.1 \mu \mathrm{L} 5 \mathrm{U} / \mu \mathrm{L}$ rTaqase (TaKaRa, Dalian, China), $2.0 \mu \mathrm{L}$ 10× rTaq Mg ${ }^{2+}$ plus buffer, $0.5 \mu \mathrm{L} 10 \mathrm{mM}$ dNTP (TaKaRa), $1.0 \mu \mathrm{L} 2.5 \mu \mathrm{M}$ primers (Sangon Biotech,

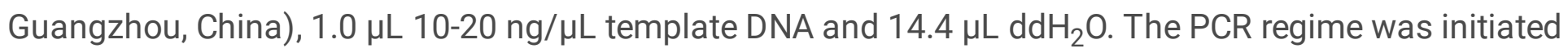
with a denaturing step $\left(94^{\circ} \mathrm{C} / 3 \mathrm{~min}\right)$, which was followed by 35 cycles of $94^{\circ} \mathrm{C} / 30 \mathrm{~s}, 50-62^{\circ} \mathrm{C} / 30 \mathrm{~s}$, $72^{\circ} \mathrm{C} / 25-30 \mathrm{~s}$ and completed with a $72^{\circ} \mathrm{C} / 5 \mathrm{~min}$ final extension. The resulting amplicons were digested for $3 \mathrm{~h}$ with the appropriate restriction enzyme (NEB Inc., Ipswich, MA, USA) at the recommended temperature in a $10 \mu \mathrm{L}$ reaction containing $1.5 \mu \mathrm{L}$ PCR product, $0.2 \mu \mathrm{L} 3 \mathrm{U} / \mu \mathrm{L}$ enzyme, $1.0 \mu \mathrm{L} 10 \times$ digestion buffer, $8.3 \mu \mathrm{L} \mathrm{ddH_{2 }}$ O. The digested amplicons were electrophoretically separated through $10-$ $12 \%$ polyacrylamide gels in the presence of Tris-boric acid-EDTA buffer run at $250 \mathrm{~V}$ for $50-60 \mathrm{~min}$.

\section{Genotyping and data analysis}

A smaller set of control cultivars, i.e., DIG, TTP, ZS97, NPB, SN265, and C039, were involved in each genotyping experiment (Table S2). The functional and nonfunctional haplotypes of each member of the Pid family were firstly determined with a couple of the functional and nonfunctional FNP markers, and new alleles were then determined with a set of allelic FNP markers by testing a regular panel consisting of each 30 representative indica and japonica type cultivars. For confirming genetic divergence of alleles between indica and japonica rice groups (if any), allele mining was then extended to two additional germplasm panels, one consisting of 40 indica type cultivars used as parents in rice breeding programs based in the southern province of Guangdong and the other of 28 japonica type cultivars used -similarly in the north-eastern province of Heilongjiang (Table S2). A $\chi^{2}$ test was used to determine whether the two genepools had or had not experienced divergence. The test was based on the formula

$$
\chi^{2}=\frac{\mathrm{N}\left[|\mathrm{ad}-\mathrm{bc}|-\left(\frac{1}{2}\right) \mathrm{N}\right]^{2}}{(\mathrm{a}+\mathrm{b})(\mathrm{c}+\mathrm{d})(\mathrm{a}+\mathrm{c})(\mathrm{b}+\mathrm{d})},
$$

where $a$ and $b$ represent the number of indica type entries scored as, respectively, harbouring or not harbouring a given allele or genotype, while $c$ and $d$ represent the same for the japonica type entries. $N$ denotes the total number of alleles or genotypes detected for each Pid gene or genotype (Fernando et al. 2018; Zhang et al. 2019; Huang et al. 2021). If all alleles derived from a given resistance gene, which was extremely diverged into indica group, then the resistance gene was defined as indica type one, and that in turn called as japonica type one.

\section{Results}

\section{Pid-2 alleles}


An alignment of Pid-2 coding sequences (CDSs) of the 15 reference cultivars revealed the presence of seven single nucleotide polymorphisms (Fig. S1). A pair of FNPs, Pid2-F/N ${ }^{\mathrm{C} 1022 \mathrm{~T}}$ and Pid2-F/N ${ }^{A 1383 G}$ effectively distinguished between the functional and the non-functional alleles. DIG, TTP, C039, ZS97 and TDK each carried a functional allele, while NPB, KSH and SN265 carried a non-functional one (Fig. 1).

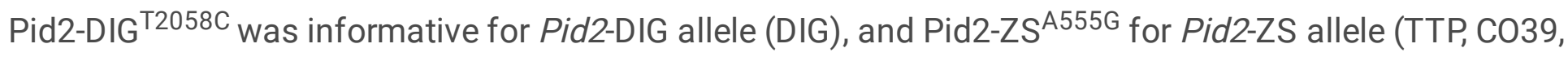
ZS97, and TDK). When the regular panel was screened, all 30 indica type entries were found to carry a functional Pid-2 allele, but this was the case for only four of the 30 japonica types (Fig. 2; Table S2). Of the 34 Pid-2 carriers, 14 belonged to Pid2-DIG, and 18 to Pid2-ZS, and two carried a distinct allele (hereafter referred to as Pid2-New). The screen of the additional 40 indica rice panel revealed that of the 39 carrying a functional copy of Pid-2, 32 had the Pid2-ZS allele, six the Pid2-DIG allele and one the Pid2New allele. None of the additional japonica germplasm panel carried a functional copy of Pid-2 (Fig. S2). A homogeneity test suggested that divergence of Pid-2 was specific to the indica genepool (Table 1). It was, therefore, defined as an indica type resistance gene.

\section{Pid-3 alleles}

The variation in the Pid-3 CDSs identified in the 15 reference cultivars comprised 29 SNPs and one indel (Fig. S3); 18 of the SNPs and the indel were targeted for marker development (data not shown). The Pid3$\mathrm{F} / \mathrm{N}^{\mathrm{G} 2009 \mathrm{~A}}$ and Pid3-F/N $\mathrm{N}^{\mathrm{C} 2209 \mathrm{~T}}$ were both effective for distinguishing between functional and nonfunctional alleles: the five cultivars DIG, TTP, C039, ZS97 and TDK each carried a functional allele, while NPB, KSH and SN265 each carried a non-functional one (Fig. 3; Table S2). Three pairs, Pid3-DIGG775A vs Pid3-DIG G2695A, Pid3-TTPC1136T vs Pid3-TTPC1623G, and Pid3-ZS ${ }^{\text {G477A }}$ vs Pid3-ZS ${ }^{\text {C525T }}$, were ensured as allele-specific FNP markers responsible for Pid3-DIG, Pid3-TTP, and Pid3-ZS, respectively (Fig. 3). As was the case for Pid-2, all 30 members of the indica panel carried a functional Pid-3 haplotype, whereas only four of the japonica panel did so (Fig. 4; Table S2). The distribution of effective alleles was highly uneven: 29 of the Pid-3 carriers harboured the Pid3-ZS allele, three the Pid3-DIG allele, one the Pid3-TTP and one a novel allele (Pid3-New). The distribution was similarly uneven in the additional indica panel, where 28 of the Pid-3 positive entries carried the Pid3-ZS allele, three the Pid3-DIG allele, one the Pid3-TTP allele and one Pid3-New; none of the members of the additional japonica panel carried an effective allele (Fig. S4). A homogeneity test implied that divergence for Pid-3 has only occurred in the indica genepool (Table 1). It was, also, referred to as an indica type resistance gene.

\section{Pid-4 alleles}

Pid-4 was by far the most diverse of the three members, with 149 SNPs and six InDels identified in the CDSs plus one intron of the 13 reference cultivars (Fig. S5), a sample of these (17 SNPs and two InDels) were targeted for marker development (data not shown). Both Pid4-F/ $\mathrm{N}^{\mathrm{C} 1217 \mathrm{G}}$ and Pid4-F/N ${ }^{\mathrm{A} 1452 \mathrm{G}}$ were informative with respect to functionality: five cultivars, DIG, NPB, KSH, C039, and SN265, were recognized as carriers of functional alleles, while TDK, TTP and ZS97 harboured non-functional alleles (Fig. 5; Table 
S2). Two pairs, Pid4-DIG ${ }^{\mathrm{A} 1149 T}$ vs Pid4-DIG ${ }^{\mathrm{A} 1898 \mathrm{G}}$, and Pid4-NPB ${ }^{\mathrm{G} 1362 \mathrm{~A}}$ vs Pid4-NPB ${ }^{\mathrm{C} 1554 \mathrm{~A}}$, were confirmed as allele-specific FNP markers responsible for Pid4-DIG and Pid4-NPB, respectively; and Pid4SN/CO ${ }^{\text {T1841A }}$ coupled with Pid4-SN/CO ${ }^{\text {G2250C }}$ responsible for both Pid4-SN and Pid4-CO (Fig. 5). Unlike the situation in both Pid-2 and Pid-3, functional Pid-4 alleles were present in many (28/30) of the japonica type entries, while the frequency of functional alleles was only moderate $(12 / 30)$ in the indica germplasm (Fig. 6; Table S2). The distribution of the various alleles was more even than was the case for Pid-2 and Pid-3, with 14 entries carrying the Pid4-SN allele, 11 the Pid4-NPB allele, eight Pid4-New and six the Pid4$\mathrm{CO}$ allele (Fig. 6). Extending the screen to the two additional panels revealed that 28/40 japonica type cultivars harboured a functional allele, while only $7 / 40$ indica type cultivars did so. Of the 35 functional haplotypes, 12 were present in entries carrying the Pid4-CO allele, 11 in those carrying the Pid4-NPB allele, seven in those carrying Pid4-New, five in those carrying the Pid4-SN allele, while just a single entry carried the Pid4-DIG allele (Fig. S6). A homogeneity test confirmed that significant divergence at Pid-4 has occurred in the japonica genepool (Table 1). Thus, it was termed 'japonica type resistance gene'.

\section{Discussion}

\section{The FNP markers for the Pid family have been comprehensively integrated}

In the present study, a deeper allele mining was carried out on the Pid family in the three panels consisting of 70 indica and 58 japonica cultivars, which were selected from various regions across landrace and modern rice eras (Tables $1 ;$ S2). Three sets of FNP markers for allele mining of the Pid family were devised based on several criterions such as representative of FNPs over a given CDS, clear genotyping, and easy-accession to users (Zeng 2020; Zhang 2020). As almost resistance genes have been initially diverged into functional and nonfunctional haplotypes (Figs. S1, S3, S5; Bryan et al. 2000; Lin et al. 2007; Liu et al. 2007; Fukuoka et al. 2009; Hua et al. 2012; Yuan et al. 2011; Zhai et al. 2011, 2014; Zhao et al. 2018; Zeng 2020), allele mining of each member of the Pid family was, therefore, initiated from haplotype differentiation with haplotype specific FNPs, which enabled us to identify any new allele in a given cultivar belonging to the functional haplotype. That in turn, allele mining could be stopped when the panel where was not any functional haplotype (Figs. S2, S4). Whereas allele mining was pursued to individual alleles with a series of allele-specific FNPs, which helped us finding out more certain alleles within the functional haplotypes. Even so, there were still 3, 2, and 14 cultivars in Pid-2, Pid3 , and Pid-4 categories, respectively, which were presumed to carry new types of alleles, compared to the defined alleles (Table 1). Collectively, the FNP markers for allele mining in the present study were largely improved from those used in the previous researches, as almost of those were focused on individual SNPs and/or InDels, which were unable to find out a series of new alleles as were the three cases shown in the current study (Shang et al. 2009; Lv et al. 2017; Promchuay and Nilthong 2017; Teerasan et al. 2019; Tian et al. 2020; Zhou et al. 2020). 


\section{The Pid family has been strictly diverged into indica and japonica subspecies}

Four alleles were detected at Pid-2, of which the three functional ones were almost entirely restricted to indica type cultivars, while the null allele was only present in japonica type germplasm (Table 1). The distribution of alleles at Pid-3 was very similar: the four functional ones were harboured for the most part by indica type entries and the null allele was common in the japonica genepool (Table 1). The latter result echoes a prior finding that the alleles of Pid-3 present in japonica type cultivars are pseudogenes (Shang et al. 2009; Lv et al. 2017). In contrast, the distribution of alleles at Pid-4 featured five functional alleles which were shared evenly among the japonica type entries, with the null allele found only in indica type ones (Table 1). It might be the first time to find out and define both indica and japonica type resistance genes within individual cultivars through allele mining with a set of comprehensive FNP markers (Table S2). The data revealed by the FNP screen suggest a plausible genetic basis for the stable and broad blast resistance exhibited by the modern cultivars, Digu, R207, Lu28S, TianfengB, R217, Zhonghua 11, Gumeizao 4, Moliruanzhan, Yuehesimiao and Yuejingsimiao 2, in that all of these cultivars harbour a functional allele at each of the three Pid genes (Table S2). It might be truly expected that integration of both indica and japonica resistance genes into upcoming cultivars would be one of the most promising ways to enlarge their genetic diversities of resistance genic resources thereby withstanding ever-growing pressure from the pathogen across indica and japonica rice areas (Shang et al. 2009; Zhang et al. 2016; Lv et al. 2017, 2020).

\section{Rather limited genotypes of the Pid family have been effective in both indica and japonica rice groups}

By owning three members of the Pid family, there would be seven all possible genotypes (combinations) $\left(2^{3}-1=7\right)$, shortly as: $d 2, d 3, d 4 ; d 2-d 3, d 2-d 4, d 3-d 4$; and $d 2-d 3-d 4$, irrespective of specific alleles. However, only three genotypes, $d 4, d 2-d 3$, and $d 2-d 3-d 4$, were detected in the three panels consisting of 128 diversified rice germplasms (Tables 1, S2). The indication is therefore that rather limited genotypes of the Pid family have been integrated into both indica and japonica rice cultivars in China. Since all the three members have been strictly diverged into the two subspecies across landrace and modern rice eras, $d 2-d 3$ was centralized in indica group and $d 4$ in japonica one both reached at overwhelming proportions; and $d 2-d 3-d 4$ also in indica group but with a rather moderate rate (Table 1). The genetic structure of the region harbouring the Pid family does not suggest any obvious barrier to local recombination (Fig. S7). That is, there were four types of such barriers in the target genomic region, the key subspecies hybrid sterile gene cluster S5 (Chen et al. 2008), the heading date gene Hd1 (Yano et al. 2000), the photonasty gene Se5 (Izawa et al. 2000), and the centromere of rice chromosome 6 (Zhao et al. 2019), all of which were enough far from the genic positions of the Pid family. In addition, genomic intervals among the three members were also enough long for independently segregation each other in a given genetic cross (Fig. S7). 
The most possible genetic determinants leading to establish such specific allelic and genotypic structures of the Pid family was, therefore, due to the specific lineage(s) of the Chinese rice population. That is, the Chinese rice population has, indeed, been derived from rather limited founder parents for an age. By reviewing the pedigrees of Top- 10 of several cultivar types including the general cultivars and $F_{1}$ hybrid crosses, Liu (2021) pointed out that the specific lineage, 'Zhenzhuai 11-ZS97', both were recognized as Pid2-ZS_Pid3-ZS, has been central to Chinese indica type rice breeding programs since the 1960s (also see www.ricedata.cn/variety). The specific lineage perfectly addressed to both questions: why there were two indica type alleles with much higher rates in the respective allelic structures, Pid2-ZS with 71.4\% (50/73), and Pid3-ZS with 86.3\% (63/73); and why there was not any single gene genotype for $d 2$ or $d 3$, but $d 2-d 3$ for being predominant among the three effective genotypes for a long time (Tables 1 , S2). That is, the unique allelic structures of the three members of the Pid family have been mainly constructed by the genotype, $d 2-d 3$, exactly, Pid2-ZS_Pid3-ZS, carried by the lineage in rice breeding programs in China since the 1960s. It was, again, concerned that updating the lineage would be the key to enlarge genetic diversities of rice cultivars in the next generation in China (Shang et al. 2009; Zhang et al. 2016; Lv et al. 2017, 2020; Liu 2021; www.ricedata.cn/variety).

A further priority will be to identify the functional specificities of the various Pid alleles by challenging near isogenic lines with a set of both indica and japonica races of the pathogen (Zhang et al. 2017, 2019; Huang et al. 2021).

\section{Conclusions}

The study has demonstrated that all the three members of the Pid family have been strictly diverged into indica and japonica subspecies: Pid-2 and Pid-3 were defined as indica type resistance genes, and Pid-4 as japonica one. Owning to the specific lineage, 'Zhenzhuai 11-ZS97', rather limited genotypes of the Pid family have been effective in both indica and japonica rice groups, of which Pid2-ZS + Pid3-ZS has been central to the Chinese rice population since the 1960s.

\section{Declarations}

\section{Author's contributions}

Project conception (QHP), Marker development (JYW, JQW, XW, QHP), Material composition (YZ, YXY, LW, $X W, J F Z, Y H Z$ ), Allele mining (RPC, JQW, XW, XMY), Data analysis (JYW, RPC, QHP), Manuscript preparation (QHP, JYW). All authors read and approved the final manuscript.

\section{Funding}

This study was supported by grants from the National Key R\&D Project (2016YDF0100601), the National Transgenic Research Project (2015ZX08001-002), and the National Natural Science Foundation of China 


\section{Availability of Data and Materials}

The data sets supporting the results of this article are included within the article and its supporting files.

\section{Ethics Approval and Consent to Participate}

Not applicable.

\section{Consent for Publication}

Not applicable.

\section{Competing interests}

The authors declare that they have no competing interests.

\section{References}

1. Bryan G, Wu K, Farrall L, Jia Y, Hershey H, McAdams S, Faulk K, Donaldson G, Tarchini R, Valent B (2000) A single amino acid difference distinguishes resistant and susceptible alleles of the rice blast resistance gene Pita. Plant Cell 12: 2033-2045

2. Chen J, Ding J, Ouyang Y, Du H, Yang J, Cheng K, Zhao J, Qiu S, Zhang X, Yao J, Liu K, Wang L, Xu C, Li X, Xue Y, Xia M, Ji Q, Lu J, Xu M, Zhang Q (2008) A triallelic system of S5 is a major regulator of the reproductive barrier and compatibility of indica-japonica hybrids in rice. Proc Natl Acad USA 195: 11436-11441

3. Chen X, Shang J, Chen D, Lei C, Zou Y, Zhai W, Liu G, Xu J, Ling Z, Cao G, Ma B, Wang Y, Zhao X, Li S, Zhu L (2006) A B-lectin receptor kinase gene conferring rice blast resistance. Plant J 46: 794-804

4. Chen Z, Zhao W, Zhu X, Zou C, Yin J, Chern M, Zhou X, Ying H, Jiang X, Li Y, Liao H, Cheng M, Li W, He M, Wang J, Wang J, Ma B, Wang J, Li S, Zhu L, Chen X (2018) Identification and characterization of rice blast resistance gene Pid4 by a combination of transcriptomic profiling and genome analysis. J. Genet Genomics 45: 665-672

5. Choi J, Purugganan M (2018) Multiple origin but single domestication led to Oryza sativa. G3 8: 797803

6. Dean R, Van Kan J, Pretorius Z, Hammond-Kosack K, Pietro A, Spanu P, Rudd J, Dickman M, Kahmann R, Ellis J, Foster G (2012) The Top 10 fungal pathogens in molecular plant pathology. Mol Plan Pathol 13: 414-430 
7. Deng Y, Zhai K, Xie Z, Yang D, Zhu X, Liu J, Wang X, Qin P, Yang Y, Zhang G, Li Q, Zhang J, Wu S, Milazzo J, Mao B, Wang E, Xie H, Tharreau D, He Z (2017) Epigenetic regulation of antagonistic receptors confers rice blast resistance with yield balance. Science 355: 962-965

8. Du H, Yu Y, Ma Y, Gao Q, Cao Y, Zhen Z, Ma B, Qi M, Li Y, Zhao X, Wang J, Liu K, Qin P, Yang X, Zhu L, Li S, Liang C (2017) Sequencing and de novo assembly of a near complete indica rice genome. Nature Commun 8: 15324

9. Ebbole D (2007) Magnaporthe as a model for understanding host-pathogen interactions. Annu Rev Phytopathol 45: 437-456

10. Fernando D, Zhang X, Selin C, Zou Z, Liban S, McLaren D, Kubinec A, Parks P, RashodH, Pamathilake R, Rong L, Yang C, Gnanesh B, Huang S (2018) A six-year investigation of the dynamics of avirulence allele profiles, blackleg incidence, and mating type alleles of Leptosphaeria maculans populations associated with canola crops in Manitoba, Canada. Plant Dis 102: 790-798

11. Flor H (1971) Current status of the gene for gene concept $₫$ Ann Rev Phytopathol 9:275-296

12. Fukuoka S, Saka N, Koga H, Ono K, Shimizu T, Ebana K, Hayashi N, Takahashi A, Hirochika H, Okuno $\mathrm{K}$, Yano M (2009) Loss of function of a proline-containing protein confers durable disease resistance in rice. Science 325: 998-1001

13. He X, Liu X, Wang L, Lin F, Cheng Y, Chen Z, Liao Y, Pan Q (2012) Identification of the novel recessive gene pi55(t) conferring resistance to Magnaporthe oryzae. Sci China Life Sci 55:141-149

14. Hua L, Wu J, Chen C, Wu W, He X, Lin F, Wang L, Ashikawa I, Matsumoto T, Wang L, Pan Q (2012) The isolation of Pi1, an allele at Pik locus which confers broad spectrum resistance to rice blast. Theor Appl Genet 125:1047-1055

15. Huang X, Zhao Y, Wei X, Li C, Wang A, Zhao Q, Li W, Guo Y, Deng L, Zhu C, Fan D, Lu Y, Weng Q, Liu K, Zhou T, Jing Y, Si L, Dong G, Huang T, Feng Q, Qian Q, Li J, Han B (2012) Genome-wide association study of flowering time and grain yield traits in a worldwide collection of rice germplasm. Nature Genetics 44: 32-39

16. Huang Z, Wang J, Zhang Y, Yao Y, Huang L, Yang X, Wang L, Pan Q (2021) Dynamics of race structures of Pyricularia oryzae populations across 18 seasons in Guangdong province, China. Plant Dis 105: 144-148

17. Izawa T, Oikawa T, Tokutomi S, Okuno K, Shimamoto K (20000) Phytochromes confer the photoperiodic control of flowering in rice (a short-day plant). Plant J 22: 391-399

18. Li C, Wang D, Peng S, Chen Y, Su P, Chen J, Zheng L, Tan X, Liu J, Xiao Y, Kang H, Zhang D, Wang G, Liu $Y$ (2019) Genome-wide association mapping of resistance against rice blast strains in South China and identification of a new Pik allele. Rice 12: 47

19. Lin F, Chen S, Que Z, Wang L, Liu X, Pan Q (2007) The blast resistance gene Pi37 encodes an NBSLRR protein and is a member of a resistance gene cluster on rice chromosome 1. Genetics 177: 18711880

20. Liu D (2021) Decoding pedigrees of the top-10 general cultivars and rice $F_{1}$ hybrid systems in China. Available via DIALOG. https://mp.weixin.qq.com/s/7L5PcYMxGFXLJIVjfSM3dQ. Accessed 23 Feb, 
21. Liu W, Wang G (2016) Plant innate immunity in rice: a defense against pathogen infection. Natl Sci Rev 3: 295-308

22. Liu X, Lin F, Wang L, Pan Q (2007) The in silico map-based cloning of Pi36, a gene encodes a coiledcoil NBS-LRR protein, conferring race-specific resistance to Magnaporthe oryzae. Genetics 176: 25412549

23. Lv Q, Huang Z, Xu X, Tang L, Liu H, Wang C, Zhou Z, Xin Y, Xing J, Peng Z, Li X, Zheng T, Zhu L (2017) Allelic variation of the rice blast resistance gene Pid3 in cultivated rice worldwide. Sci Rep 7: 10362

24. Lv Q, Li W, Sun Z, Ouyang N, Jing X, He Q, Wu J, Zheng J, Zheng J, Tang S, Zhu R, Tian R, Duan M, Tan Y, Yu D, Sheng X, Sun X, Jia G, Gao H, Zeng Q, Li Y, Tang L, Xu Q, Zhao B, Huang Z, Lu H, Li N, Zhao J, Zhu L, Li D, Yuan L, Yuan D (2020) Resequencing of 1,143 indica rice accessions reveals important genetic variations and different heterosis patterns. Nature Commun 11: 4778

25. Ouyang Y, Zhang Q (2013) Understanding reproductive isolation based on the rice model. Annu Rev Plant Biol 64: 111-135

26. Promchuay A, Nilthong S (2017) Investigation of Pid3 rice blast resistance gene in northern upland rice varieties (Oryza sativa L.), Thailand using molecular markers. J Advanced Agri Technol 4: 209214

27. Shang J, Tao Y, Chen X, Zou Y, Lei C, Wang J, Li X, Zhao X, Zhang M, Li Z, Xu J, Cheng Z, Wang J, Zhu $L$ (2009) Identification of a new rice blast resistance gene, Pid3, by genome-wide comparison of paired nucleotide-binding site-leucine-rich repeat genes and their pseudogene alleles between the two sequenced rice genomes. Genetics 182: 1303-1311

28. Teerasan W, Srikaew I, Phaitreejit K, Kate-Ngam K, Jantasuriyarat C (2019) Gene-specific marker screening and disease reaction validation of blast resistance genes, Pid3, Pigm and Pi54 in Thai landrace rice gerplasm and recommended rice varieties. Plant Genetic Resources: Characterization and Utilization 17: 421-426

29. Tian D, Lin Y, Chen Z, Chen Z, Yang F, Wang F, Wang Z, Wang M (2020) Exploring the distribution of blast resistance alleles at the Pi2/9 locus in major rice-producing areas of China by a novel Indel marker. Plant Dis 104: 1932-1938

30. Wang X, Jia Y, Wamishe Y, Jia M, Valent B (2017) Dynamic changes in the rice blast population in the USA over six decades. Mol Plant-Microbe Interac 30:803-812

31. Wang W, Mauleon R, Hu Z et al. (2018) Genomic variation in 3,010 diverse accessions of Asian cultivated rice. Nature 557: 43-49

32. Wu W, Wang L, Zhang S, Li Z, Zhang Y, Lin F, Pan Q (2014) Stepwise arms race between AvrPik and Pik alleles in the rice blast pathosystem. Mol Plant-Microbe Interac 27:759-769

33. Xiao G, Yang J, Zhu X, Wu J, Zhou B (2020) Prevalence of ineffective haplotypes at the rice blast resistance $(R)$ gene loci in Chinese elite hybrid varieties revealed by sequence-based molecular diagnosis. Rice 13: 6 
34. Yano M, Katayose Y, Ashikari M, Yamanouchi U, Monna L, Fuse T, Baba T, Yamamoto K, Umehara Y, Nagamura Y, Sasaki T (2000) Hd1, a major photoperiod sensitivity quantitative trait locus in rice, is closely related to the Arabidopsis flowering time gene CONSTANS. Plant Cell 12: 2473-2483

35. Yuan B, Wang L, Zeng X, Xu X, Zhai C, Hu H, Lin F, Pan Q (2011) The Pik-p resistance to Magnaporthe oryzae in rice is mediated by a pair of closely linked CC-NBS-LRR genes. Theor Appl Genet 122: 10171028

36. Zeigler R, Cuoc L, Scott R, Bernardo M, Chen D, Valent B, Nelson R (1995) The relationship between lineage and virulence in Pyricularia grisea in the Philippines. Phytopathology 85:443-451

37. Zeng $S$ (2020) Identification of rice blast resistance genes in the key parental cultivars. Dissertation, South China Agri University, Guangzhou, China (In Chinese with English Abstract)

38. Zhai C, Wang L, Dong Z, Zeng X, Lin F, Pan Q (2011) The rice blast resistance gene, Pik, is the third allele at the Pik cluster that is distinguished by a function-associated SNP. New Phytologist 189: 321334

39. Zhai C, Zhang Y, Yao N, Lin F, Liu Z, Dong Z, Wang L, Pan Q (2014) Function and interaction of the coupled genes responsible for Pik-h encoded rice blast resistance. Plos One 9: e98067

40. Zhang J, Chen L, Xing F, Kudrna D, Yao W, Copetti D, mu T, Li W, Song J, Xie W, Lee S, Talag J, Shao L, An Y, Zhang C, Ouyang Y, Sun S, Jiao W, Lv F, Du B, Luo M, Maldonado C, Goicoechea J, Xiong L, Wu C, Xing Y, Zhou D, Yu S, Zhao Y, Wang G, Yu Y, Luo Y, Zhou Z, Hurtado B, Danowitz A, Wing R, Zhang $Q$ (2016) Extensive sequence divergence between the reference genomes of two elite indica rice varieties Zhenshan 97 and Minghui 63. Proc Natl Acad Sci USA E5163-5171

41. Zhang R (2020) Resistance gene composition of differential cultivars for rice blast pathosystem. Dissertation, South China Agri University, Guangzhou, China (In Chinese with English Abstract)

42. Zhang S, Wang L, Wu W, He L, Yang X, Pan Q (2015) Function and evolution of Magnaporthe oryzae avirulence gene AvrPib responding to the rice blast resistance gene Pib. Sci Rep 5:11642

43. Zhang Y, Zhu Q, Yao Y, Zhao Z, Correll J, Wang L, Pan Q (2017) The race structure of the rice blast pathogen across southern and northeastern China. Rice 10: 46

44. Zhang Y, Wang J, Yao Y, Jin X, Correll J, Wang L, Pan Q (2019) Dynamics of race structures of the rice blast pathogen populations in Heilongjiang province, China from 2006 through 2015. Plant Dis 103: $2759-2763$

45. Zhao H, Wang X, Jia Y, Minkenberg B, Wheatley M, Fan J, Jia M, Famoso A, Edwards J, Wamishe Y, Valent B, Wang G, Yang Y (2018) The rice blast resistance gene Ptr encodes an atypical protein required for broad-spectrum disease resistance. Nature Commun 9: 2039

46. Zhao L, Wang S, Cao Z, Ouyang Y, Zhang Q, Xie L, Zheng R, Guo M, Ma M, Hu Z, Sung W, Zhang Q, Li G, Li X (2019) Chromatin loops associated with active genes and heterochromatin shape rice genome architecture for transcriptional regulation. Nature Commun 10: 3640

47. Zhou Y, Lei F, Wang Q, He W, Yuan B, Yuan W (2020) Identification of novel alleles of the rice blastresistance gene $P i 9$ through sequence-based allele mining. Rice 13: 80 


\section{Table}

Due to technical limitations, table 1 is only available as a download in the Supplemental Files section.

\section{Figures}

a

$\operatorname{Pid} 2-\mathrm{F} / \mathrm{N}^{\mathrm{C} 1022 \mathrm{~T}}$

$(109 / 85 \mathrm{bp})$

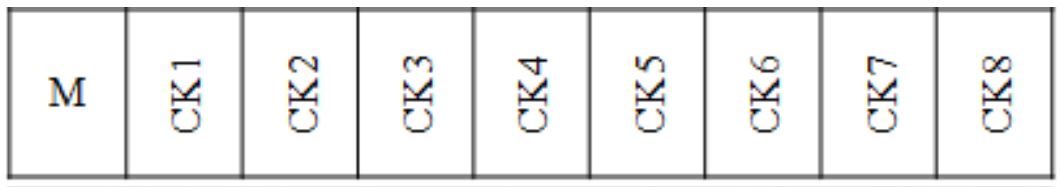

$\mathrm{Pid} 2-\mathrm{F} / \mathrm{N}^{\mathrm{A} 1383 \mathrm{G}}$

$(126 / 104 \mathrm{bp})$
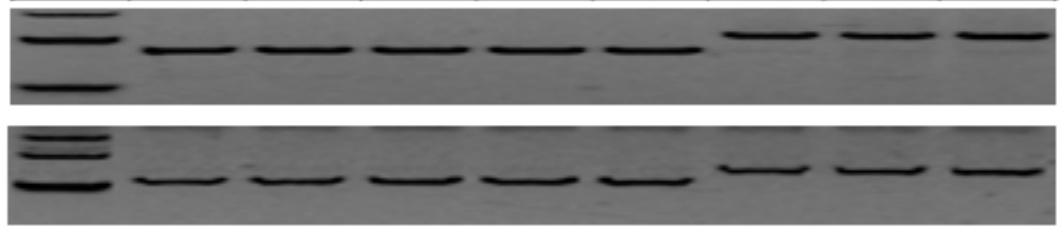

b

Pid2-DIG ${ }^{\text {T2058C }}$ $(226 / 174 \mathrm{bp})$

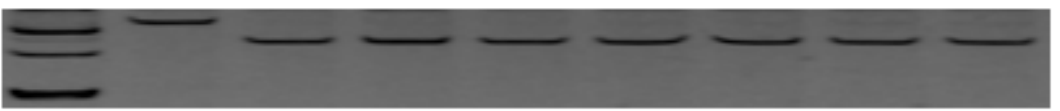

c

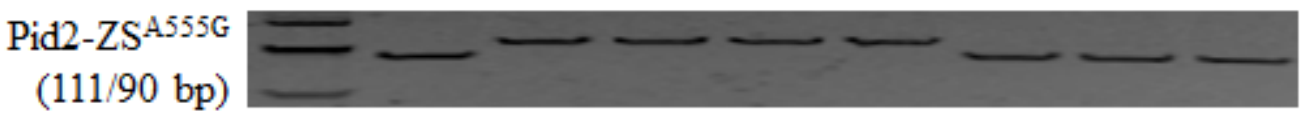

\section{Figure 1}

Development of a set of FNP markers able to distinguishing both haplotypes and alleles of Pid-2. (a-c) Discriminating between functional $(\mathrm{F})$ and non-functional $(\mathrm{N})$ haplotypes and between Pid2-DIG and Pid2-ZS alleles. CK1, Digu (Pid2-DIG); CK2, Tetep (Pid2-ZS); CK3, CO39 (Pid2-ZS); CK4, ZS97 (Pid2-ZS); CK5, Taluka (Pid2-ZS); CK6, Nippon bare (Pid2-Null); CK7, Kochukaru (Pid2-Null); CK8, Shennong265 (Pid2-Null). M, DL-500 size marker.

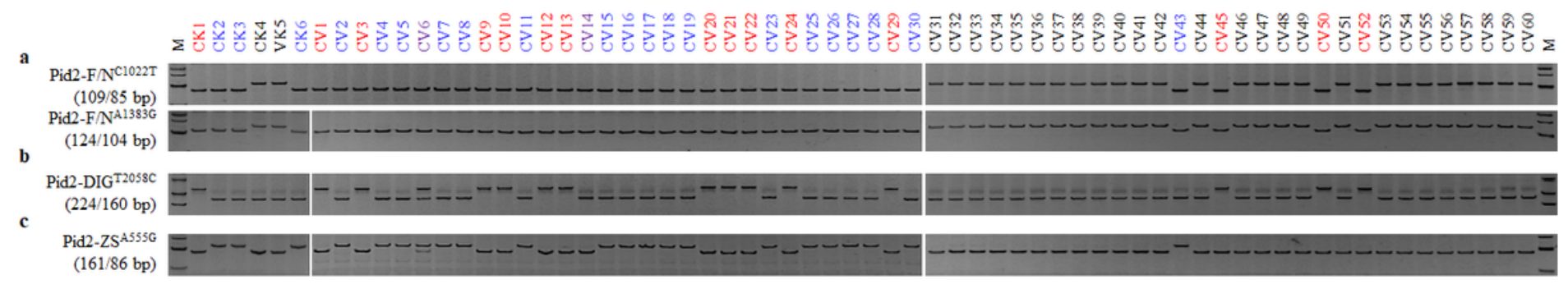

\section{Figure 2}

Alleles of Pid-2 represented in the regular panel consisting of both indica (CV1-30) and japonica (CV3160) types. (a) Functional and non-functional haplotypes, (b) the Pid2-DIG allele, (c) the Pid2-ZS allele. CK1, Digu (Pid2-DIG; red); CK2, Tetep (Pid2-ZS; blue); CK3, ZS97 (Pid2-ZS; blue); CK4, Nipponbare (Pid2Null; black); CK5, Shennong265 (Pid2-Null; black); CK6, CO39 (Pid2-ZS; blue); and the undefined alleles carried by CV6 and CV14 was marked in purple. The detailed information on each entry was shown in Table S2. M, DL-500 size marker. 
a

\begin{tabular}{|c|c|c|c|c|c|c|c|c|}
\hline $\mathrm{M}$ & $\bar{U}$ & $\frac{U}{U}$ & $\stackrel{M}{U}$ & $\stackrel{v}{U}$ & $\frac{n}{3}$ & $\frac{1}{0}$ & $\frac{1}{3}$ & $\ddot{U}$ \\
\hline
\end{tabular}

\section{Pid3-F/N ${ }^{\mathrm{G} 2009 \mathrm{~A}}$ \\ (138/101 bp) \\ Pid3-F/N $\mathrm{N}^{\mathrm{C} 2209 \mathrm{~T}}$ \\ (216/145/71 bp)}

b

$$
\begin{array}{r}
\text { Pid3-DIG }{ }^{\text {G775A }} \\
(138 / 102 \text { bp }) \\
\text { Pid3-DIG } 2695 \mathrm{~A} \\
(148 / 121 \mathrm{bp})
\end{array}
$$

c
Pid3-TTP C1136T
(110/85 bp)
Pid3-TTPC1623G
(120/102 bp)
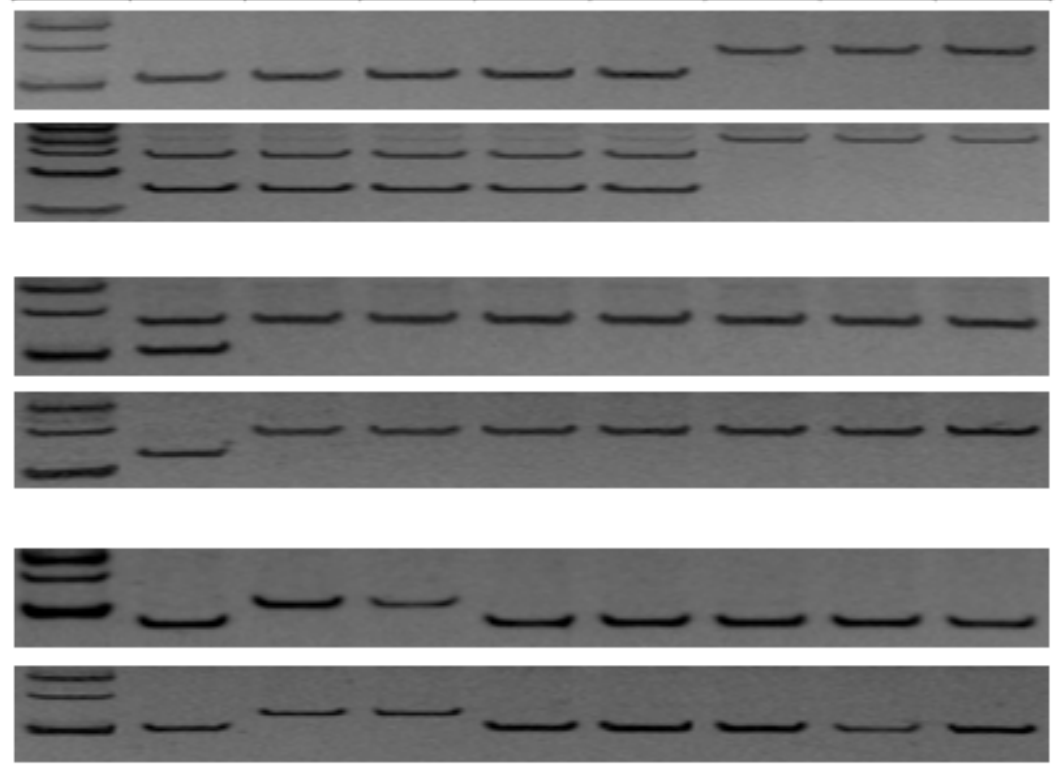

d

Pid3-ZSG477A (145/125 bp)

Pid3-ZSC525T (125/104 bp)

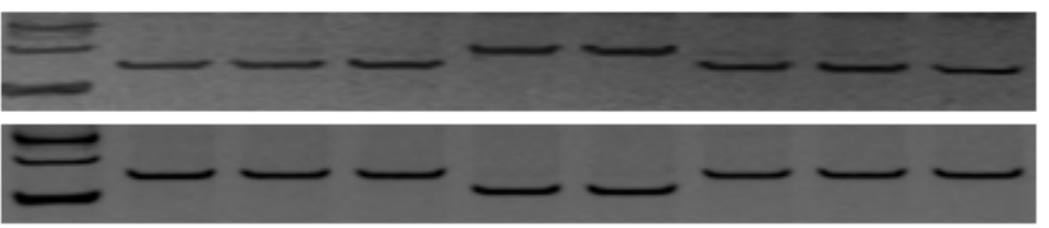

\section{Figure 3}

Development of a set of FNP markers able to distinguishing both haplotypes and alleles of Pid-3. (a-d) Discriminating between functional $(\mathrm{F})$ and non-functional $(\mathrm{N})$ haplotypes and among Pid3-DIG, Pid3-TTP, and Pid3-ZS alleles. CK1, Digu (Pid3-DIG); CK2, Tetep (Pid3-TTP); CK3, Tadukan (Pid3-TTP); CK4, ZS97 (Pid3-ZS); CK5, C039 (Pid3-ZS); CK6, Nipponbare (Pid3-Null); CK7, Shennong265 (Pid3-Null); CK8, Koshihikari (Pid3-Null). M, DL-500 size marker.

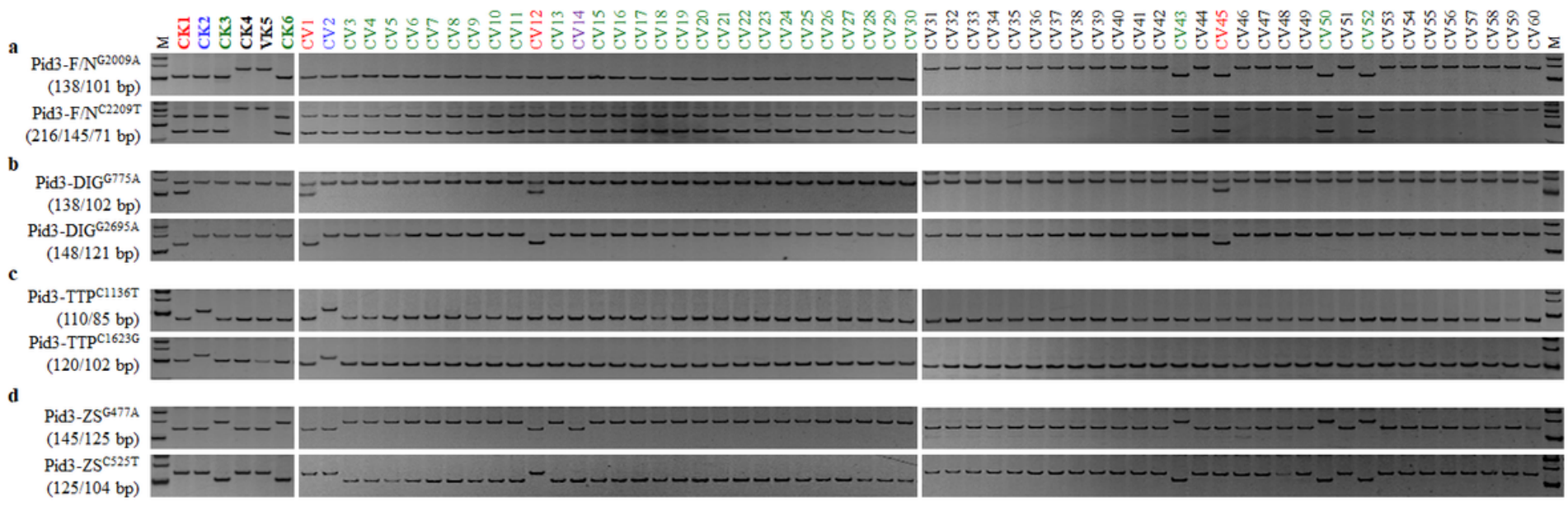

Figure 4 
Alleles of Pid-3 represented in the regular panel consisting of both indica (CV1-30) and japonica (CV3160) types. (a) Functional and non-functional haplotypes, (b) the Pid3-DIG allele, (c) the Pid3-TTP allele, (d) the Pid3-ZS allele. CK1, Digu (Pid3-DIG; red); CK2, Tetep (Pid3-TTP; blue); CK3, ZS97 (Pid3-ZS; green); CK4, Nipponbare (Pid3-Null; black); CK5, Shennong265 (Pid3-Null; black); CK6, C039 (Pid3-ZS; green); and the undefined allele carried by CV14 was marked in purple. The detailed information on each entry was shown in Table S2. M, DL-500 size marker.

a

$$
\begin{array}{r}
\text { Pid4-F/N }{ }^{\mathrm{C} 1217 \mathrm{G}} \\
(107 / 73 \mathrm{bp}) \\
\text { Pid4-F/N } \\
(115 / 97 \mathrm{bp})
\end{array}
$$

\begin{tabular}{|c|c|c|c|c|c|c|c|c|}
\hline $\mathrm{M}$ & $\bar{U}$ & $\stackrel{v}{U}$ & 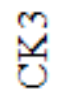 & $\underset{U}{\forall}$ & 光 & $\ddot{v}$ & $\sqrt{0}$ & $\begin{array}{l}\infty \\
\mho \\
ن\end{array}$ \\
\hline
\end{tabular}

b

c

$$
\begin{array}{r}
\text { Pid4-DIGA1149T } \\
(160 / 136 \text { bp }) \\
\text { Pid4-DIGA1898G } \\
(114 / 72 \text { bp) }
\end{array}
$$
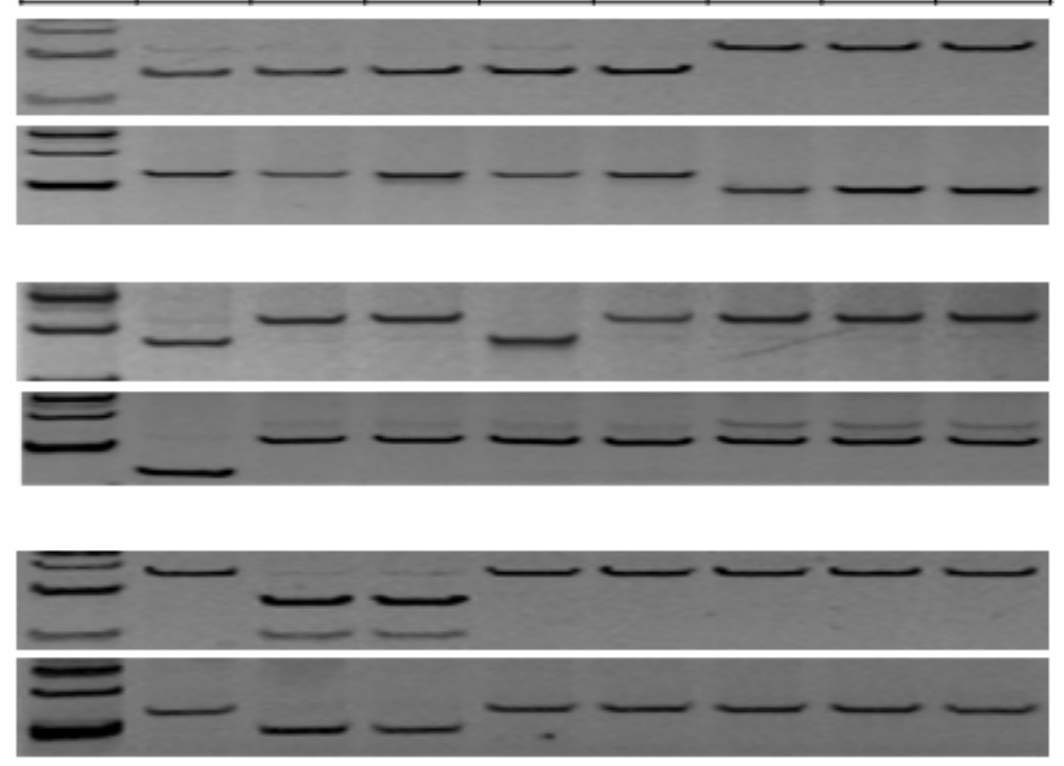

d

NPB

(130/81 bp)

Pid4-NPB ${ }^{\mathrm{C} 1554 \mathrm{~A}}$

(118/97 bp)

\section{Pid4-SN/COT1841A \\ (195/161 bp)}

Pid4-SN/COG2250C

(120/92 bp)

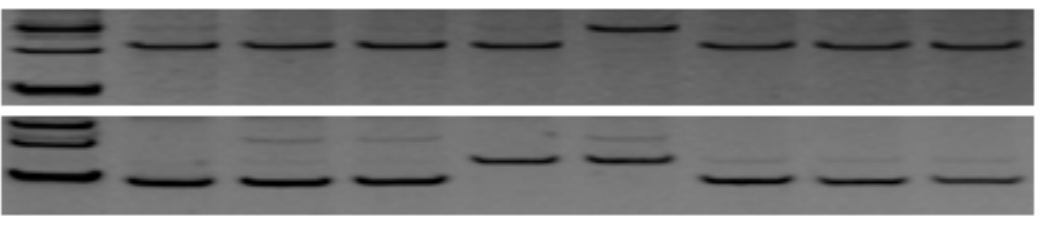

\section{Figure 5}

Development of a set of FNP markers able to distinguishing both haplotypes and alleles of Pid-4. (a-d) Discriminating between functional $(F)$ and non-functional $(N)$ haplotypes and among Pid4-DIG, Pid4-NPB, Pid4-CO, and Pid4-SN alleles. CK1, Digu (Pid4-DIG); CK2, Nipponbare (Pid4-NPB); CK3, Koshihikari (Pid4NPB); CK4, CO39 (Pid4-CO); CK5, Shennong265 (Pid4-SN); CK6, Tadukan (Pid4-Null); CK7, Tetep (Pid4Null); CK8, ZS97 (Pid4-Null). M, DL-500 size marker. 


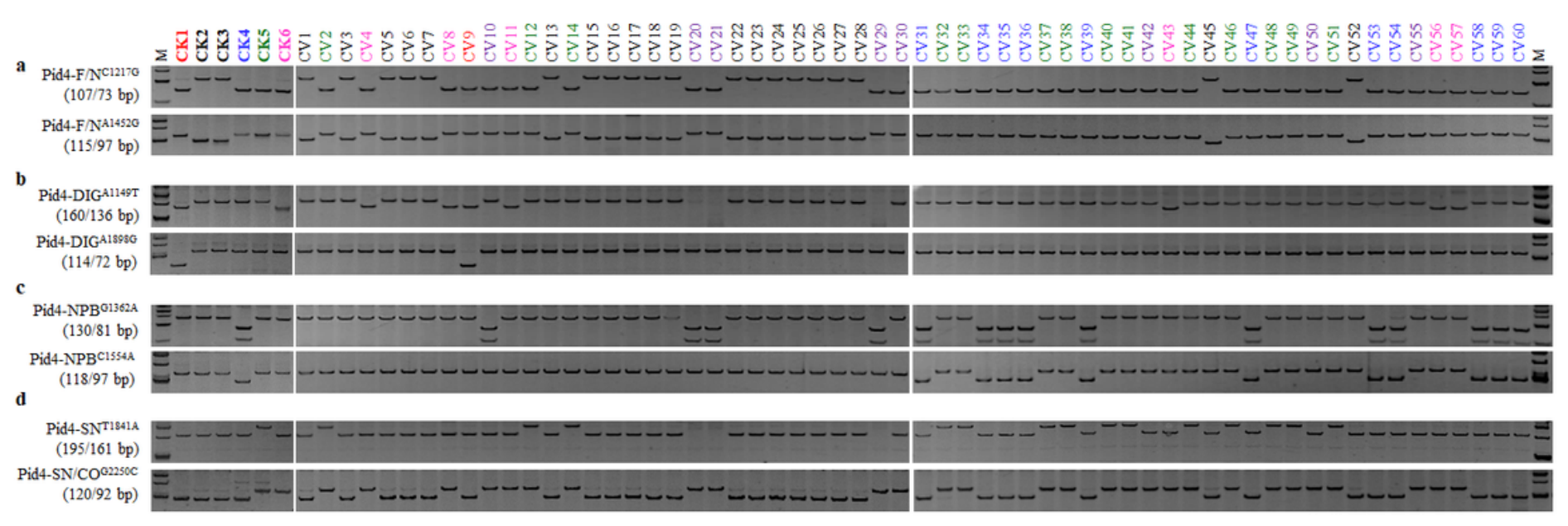

\section{Figure 6}

Alleles of Pid-4 represented in the regular panel consisting of both indica (CV1-30) and japonica (CV3160) types. (a) Functional and non-functional haplotypes, (b) the Pid3-DIG allele, (c) the Pid3-NPB allele, (d) the Pid4-SN and Pid4-CO alleles. CK1, Digu (Pid4-DIG; red); CK2, Tetep (Pid4-Null; black); CK3, ZS97 (Pid4-Null; black); CK4, Nipponbare (Pid4-NPB; blue); CK5, Shennong265 (Pid4-SN; green); CK6, C039 (Pid4-CO; rose-red); and the undefined alleles carried by CVs 10, 20, 21, 29, 30, 42, 50, and 55, were marked in purple. The detailed information on each entry was shown in Table S2. M, DL-500 size marker.

\section{Supplementary Files}

This is a list of supplementary files associated with this preprint. Click to download.

- TableS1.Markerinfo.docx

- Tables2.AlistofcvsNew.xlsx

- Fig.S1.Pid2CDS.pptx

- Fig.S2.Pid2identitiesinGDHLJ.pptx

- Fig.S3.Pid3CDS.pptx

- Fig.S4.Pid3identitiesinGDHLJ.pptx

- Fig.S5.Pid4CDS.pptx

- Fig.S6.Pid4identitiesinGDHLJ.pptx

- Fig.S7.Haplotypestructures.pptx

- Table1.png 